\title{
COMPLICACIONES OSTEOARTICULARES EN LA FIEBRE TIFOIDEA
}

\author{
Por el Dr. ALFREDO RAIMANN \\ Hospital M. Arriarán. Cátedras de Pediatria de los Profs. A. Baeza Goñi y Julio Meneghello.
}

La fiebre tifoidea, en su carácter de afección septicémica, suele comprometer el sistema esquelético, originando lesiones osteoarticulares, que se traducen en alteraciones clínico-radiológicas.

Boerhave ${ }^{\sharp}$ en 1728 , destaca por primera vez la existencia de complicaciones óseas en la fiebre tifoidea. Posteriormente, Maissoneuye ${ }^{8}$, en 1833, y Chaissaignac ${ }^{\S}$, en 1854, aumentan el caudal de estas obseryaciones y atraen sobre esta materia la inquietud de clínicos y de bacteriólogos.

El descubrimiento del bacilo de Eberth, en 1880 , proporcionó una base de mayor precisión en el estudio de las complicaciones esqueléticas de la fiebre tifoidea. La investigación señaló con cierta frecuencia la supuración en las lesiones óseas, fenómeno que no ocurre cuando el germen tífico se localiza en otros órganos.

En 1890 demostró Colzis, experimentalmente, el poder piógeno del bacilo aislado del pus de un absceso tibial, desarrollado en una recaída de fiebre tifojdea. Después de cultivar el germen, este autor lo inyectó por vía endovenosa a una serie de 14 conejos, a los que le fracturó el fémur algunos minutos más tarde. En 11 de los animales comprobó Ia formación de abscesos en el lugar del traumatismo, aislando de ellos sólo el B. de Eberth.

Muschko", en esa época observó un caso de osteítis costal, que se prolongó durante 7 años, encontrándose en esta oportunidad el bacilo tífico. Con posterforidad se llegó a demostrar plenamen- te el origen septicémico de las complicaciones osteoarticulares.

Las complicaciones esqueléticas de orden tífico se traducen desde un punto de vista general, en lesiones variables, que fundamentalmente permiten catalogarse como osteítis o periostitis dolorosas, o bien como osteoartritis, a veces deformantes y que pueden conducir a fenómenos de luxación patológica. La localización de las lesiones óseas es muy variable. Asi Murphy ${ }^{3}$, haciendo una recopilación, encontró que de 553 complicaciones óseas, 110 correspondian a las vértebras, 22 a los huesos craneanos, 142 al tórax, 57 a las extremidades superiores, 83 a las extremidades inferiores y 19 a localizaciones varias.

La frecuencia ha sido estimada en 0.45 y $0.87 \%$ por Blake y Mason y de 0.8 por Murphy y Ross Veal'.

Se presentan frecuentemente en la convalecencia, aunque a veces pueden manifestarse mucho tiempo después, ya que el $B$. de Eberth tiene una supervivencia muy grande en el organismo. Cormil, Chantemesse y Widal, Richet y Piquet $^{s}$ han señalado osteoperiostitis aparecidas hasta 15 años después de la enfermedad causal. Murphy demostró el bacilo en un absceso, 20 años después de la infección. Jaureguy y $\operatorname{Simon}^{11}$, de Uruguay, ayudados del mielocultivo, obtienen resultados positivos en la búsqueda del germen, hasta el día 54 después del comienzo de la enfermedad.

Las complicaciones osteoarticulares en la fiebre tifoidea ocurren de preferencia en el niño y en el adulto jo- 
ven. S6lo en forma excepcional han sido vistas en enfermos mayores de 25 años. Pueden ser originadas por el $B$. de Eberth o por cualquier otro germen de la supuración. En ocasiones hay asociaciones de gérmenes. Estas lesiones revisten distinto aspecto $\mathrm{y}$ gravedad $\mathrm{y}$ su evolución puede ser aguda, subaguda o crónica.

\section{Material.}

En el plazo de 7 años se han atendido 684 casos de fiebre tifoidea en el Hospital Manuel Arriarán, de los que se complicaron con lesiones óseas o articulares o ambas a la vez con francas alteraciones radiológicas, 8 . Ha habido 6 enfermos en los que clínicamente se sospechó la existencia de una complicación ósea o articular; pero que no fué confirmada por la radiología. En otros 9 enfermos hemos observado sólo dolor a nivel de un miembro o a nivel de la región costal y que se interpretó de distinta manera, ya como neuritis o ya como osteítis. En algunos enfermos se comprometieron 2 ó más regiones en forma sucesiva.

Ninguno de los pacientes que presentaron complicaciones osteoarticulares recibió cloromicetina.

\section{Casuística.} sal.

GASO N"1. - Espondilitis de la regiŕn dorI. T. O. - Oibs, N2 $47 / 33457$.

corresponde a una enferma de 12 años, con aracnodactilia, que ingresa al Hospital el 4-II-1947 por una fiebre tifoidea de 8 días de evolución $y$ confirmada mediante el aislamiento del B. de Eberth de las deposiciones y del pus de un absceso de la parótida.

Este caso se caracterizó por la extraordinaria gravedad, evidenciada en la evolución prolongada, con gran compromiso general, estado tífico intenso, tendencia hemorragipara clara, que se tradujo en frecuentes epistaxis, que requịileron cauterización; en la melena que se prolongo por espacio de una semana $y$ en el síndrome purpúrico de predominio en las extremídades $y$ tronco. En la segunda semana de hospitalización se presentó una parotiditis bilateral, que a derecha precisó de la evacuación quirúrgica, aislándose del pus el $B$. de Eberth.
La temperatura fué irregular y elevada durante 8 dias; luego persiste por 12 días más; pero en forma arrastrada. Después de 3 dias de apirexia, ze instala un nuevo proceso febril, que dura 89 días. Este cuadro se interpretó como una probable recaida. A los 60 dias de hospitalización (ic6 je enfermedad) se aprecia un agravamiento del estado general, fiebre de $39^{\circ}$ y dolores en las regiones zacra e iliaca, que se exacervaban con la presión. Pasan transitorlamente al $5^{\circ}$ dia de haber comenzado, para reaparecer al siguiente, referidos a la cadera izquierda, a partir de la región sacra. A los 70 días de hospitalizactón se observa una nueva alzą térmica. Persiste el dolor a la presión de fa articulación sacrolumbar. Se torna ese día la radiograíía $N^{n}$ 62273, cuyo informe es el siguiente: "Los cuerpos de las vértebras lumbares 3 y 4 se observan irregularmente contrastadas; pero esto puede ser originado por la superposición de las sombras del colon". Fn los días sucesivos perisiste el dolor encontrado en los exámenes anteriores y a los 18 dias de haber comenzado se advierte actitud de defensa con flexión de la pierna y muslo $\mathrm{y}$ aducción $\mathrm{y}$. rotación interna del miembro izquierdo. Además, habia contractura muscular y dolor a la presión de la 12 vértebra dorsal y 1: lumbar. Días más tarde se le coloca corset de yeso, con to que dismlnuyen rápldamente lus dolores, desapareciendo totalmente a los 93 días de hospitalización. A los 3 meses de haber sido colocado, se retịa el yeso, existiendo en ese momento aún rigidez cle la columna y una pequeña cifosis a nivel de las vértebras dorsales 10 y 11 . Siete dias más tarde se toma la radiografía $\mathrm{N}^{\circ}$ 64862. que señala una gran disminución del espacio intervertebral entre las vertebras dorsales 11 y 12 .

Resumen y comentario: Este caso corresponde a una espondilitis tífica, que se presentó durante la recaída de una fiebre tifoidea grave. Este enfermo tuvo un sindrome doloroso. espinal, que se acompañó de alteraciones radiológicas claras, consistentes en un acortamiento del espacio intervertebral comprendido entre las vértebras dorsales 11 y 12 . Se caracterizó esta complicación por su benignidad, hecho que señala la literatura. Se debería esto a la época en que aparece, ya que lo hace generalmente en un momento en que. el organismo cuenta con defensas humorales importantes. Gall $^{3}$ estima que un caso de cada 1,800 fiebres tifoideas se complica de espondilitis, habiendo un predominio de los hom- 
bres sobre las mujeres $(83 \%)$. El pro nóstico es favorable. Se han observado recidivas, pero son raras. La curación es lenta y puede durar varios meses, terminando casi siempre con la ánquilosis del segmento afectado, por lo que queda una limitación de la movilidad.

Tratamiento: Se aconseja reposo en cama dura. Cuando el dolor y la contractura han disminuído hay que realizar movilización cou corset enresado.

ICASO N"2, - Osteoartritis de la muñeca izquierda.

S. M. A. - Obs. N. 49/56637.

Se refiere a un niño de 12 años, que ingresa al Hospital el 11-V-1949 por una fiebre tifoidea que llevaba 11 días de evolución y que se confirmó con la positividad de las reacciones de aglutinación a altas tasas. Se trató de una forma muy grave, que hizo una evolución muy accidentada y con múltiples complícaciones. Entre éstas figuran una miocarditis, un purrura tombopénico desde el $7 \%$ dfa y una estomatitis máculofibrinoza a los 23 coías de hospitalización. Presentó, además, edemas carenciales.

A los 27 dias de hospitalizazlión (34 de enfermedad) se aprecla un aumento de volumen doloroso, sin cambio de coloración, a nivel de la muñeca jzquierda. Al día siguiente se toma una radiografia cuyo informe señala: "Discreta rarefacción ósea de la metáfisis distal del cúbito". Dos días más tarde existe edema bastante considerable y la palpacion revela dolor en el extremo distal del cúbito. En los dias suresivos se nota una regresión del proceso. A los $\mathbf{3 4}$ días de hospitalízación (1.1 de haber comenzado la complicación) se toma una nueva radiografía que informa: "Aumento de las sambras de las partes blandas de la región radiocarpiana. Osteoporosis de los huesos del carpo con idismintución de la nitidez de los contornos de ellos". Al dia siguiente se nota reagravación del proceso; existia otra vez calor local y discreto cambio de coloración, acompañados de impotencia funcional relativa, por lo que se punclona la articulacicn, cobtenténdose 1 cc de líquido opalescente, cuyo acultivo dió desarrollo de B. de Eberth de tipo vi.

El proceso inflamatorio se mantuvo sin vartar hasta los 189 días de hospitalización, momento en el cual se aprecia una franca reducción del aumento de volumen de la cara dorsal de la muñeca; pero persiste siempre el dolor a la palpación de la interlínea articular por su cara palmar. Siete dias más tarde hay aún escaso folor a nivel de la lnterlinea articular $y$ que desaparecló 2 días después. Se tomaron dos nuevas radiografías de control, una de ellas (N 71315) a los 45 dias de hospitalización, en un momento en que estaba en plena evolución el proceso articular y en la que se camprobó un pequeño defecto óseo del semilunar de la mano izquierda. Diez días después de haber mejorado elínicamente la artritis, se tomó una última radiografía $\left(N^{\circ} T^{\prime} 1749\right)$, en la que se observan algunas zonas de osteoesclerosis dentro del semllunar.

El tratamiento consistió en inmovilización con valva de yeso y vendaje. Posteriormente se hizo movilización pasiva $\mathrm{y}$ luego activa. La recuperación fué completa.

Resumen: Se trató de una fiebre tifoidea muy grave y con múltiples complicaciones. Una de ellas fué la osteoartritis de la muñeca izquierda, que corresponde a una localización rara dentro de este tipo de complicaciones producidas por el B. de Eberth. Se presentó en plena convalecencia de la enfermedad. La etiología precisa pudo ser determinada mediante la punción del proceso articular. Su evolución fué benigna y no dejó secuelas.

CASO N: 3. - Osteoartritis de la articulación coxofemoral izquierda.

A. A. A. - Obs. $\mathbb{N}^{*} 46 / 12206$.

Niña de 6 años de edad, que ingresa al Hospital el 19-VII-1946 por una fiebre tifojdea que llevaba 20 dias de evolución. A los 5 dias de haber comenzado el cuadro infeccioso, acusa dolor permanente a njvel de la cadera y rcdilla tzoulerdas y 4 dias antes de consultar en el hospital, observa la madre aumento de volumen de estas articulaciones. El examen físico de ingreso mostró una niña muy pálida, enflaqueclda y con discreta clanosis de los labios y uñas. Tenía hepato y espienomegalia. Las articulaciones coxofemoral, rodilla y tiblotarsiana izquierdas estaban aumentadas de volumen. dolorosas, con coloración violácea y con calor local.

Al ingreso se plantean las hipótesis diagnóstica de septicemta o enfermedad reumátlca. Sin embargo. la positividad de las reacclones de aglutinación a títulos elevados en forma repetida, unido a los hallazgos clínicos. inclinó el diagnóstito hacia una fiebre tlfoidea.

A los 2 días de hospitalizactión se observa un aumento de volumen difuso del muslo izqulerdo $₹$ que era especialmente notorio a nivel de su parte ajta. Tres días más tard? se coloca el miembro inferior en extenstón continua. con lo que pasan las molestias subjetivas. A los 20 dias de estadía en el hosvital se ralna el fémur muy aumentado ds volumen; pero aparentemente algo disml- 
nuido con respecto a los exámenes anteriores.

Fl 21-TX-46 sę traslada a Cirugía, en donde se le toma una radiografia, cuyo informe es el siguiente: "Osteomielitis de todo el fémur izqulerdo, cuya diafisis forma un gran secuestro envuelto en su casi totalidad por hueso periostal de neoformación. Hay una luxación de la cadera de este lado. "Ese día se coloca una tracción con estribo. El 12-XI-46. o sea, a los 103 días de haber comenzada la enfermedad, se toma una nueva radiografía, cuyo Informe es: "Osteomielitis de toda la diáfisis $y$ epífisis superior del fémur izquierdo. En comparación con la radografía anterior se observa una reabsorción casi total de la epifisis superior, a la vez que una regeneración acentuada de la diáfisis". Dos días más tarde se coloca un yeso pelvipedio.

6-III-47: Se retira el yeso. Se ahserva una deformación en el contorno de la cadera. La movilidad pasiza era poco dolorosa; la actipa estaba muy limitarda. Una radiografía de control revela todavía actividad del proceso, por lo que se coloca nuevo yeso por 2 meses.

20-V-47: Se aprecla el miembro en posiclón satisfactorla. Hay cierto grado de movilidad do la cadera, pero que es dolorosa. Rodilla con movilidad muy limitada $y$ dolorosa. Fémur muy engrosado, pero indoloro. No se aprecia gran aumento de volumen del musslo. La radiografia $\mathrm{N}^{*} 60048$ revela destrucclón completa. de la cabeza femoral. Sólo se aprecla el cuello, que se apoya en la parte superior de la ceja cotiloídea. Hay regeneración casi completa del fémur y desaparición del secuestro cbservado anteriormente. Se coloca nuevo yeso.

19-1X-47: Abierto el yeso en valva se encuentra que persiste la movilidad de la cadera en flexoextensión. Rodllla izquierda siem. pre con movilidad muy limitada. Longitud espino maleolar: $51.5 \mathrm{~cm}$ a izquierda; $53.5 \mathrm{~cm}$ a derecha.

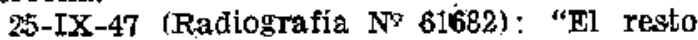
que queda del cuello anatómico (1/3 externo) aparece encajado en un pequeño neocotilo que se ha formado en la parte superior del cotilo. El núcleo del trocánter mayor simula una cabeza".

Una última radlografía tomada algunos meses más tarde, muestra un progreso hacia la regeneración total de la díficis femoral.

Resumen: Este caso corresponde a una artritis de la articulación coxofemoral izquierda, con osteomielitis del fémur, formación de un gran secuestro, reabsorción de la epifisis superior y luxación de la cadera. Se discutió mucho la etiología del proceso. Pensamos que este caso corresponde más bien a una osteomielitis y artritis por germen piógeno del tipo del estafilococo. El hemocultivo practicado resultó negativo y desgraciadamente no se practicó una punción de la articulación.

A pesar de la tracción e inmovilización tuvo una evolución muy arrastrada, debiendo prolongrase ésta por espacio de 1 año 2 meses. Como secuela quedó un acortamiento del miembro inferior izquierdo.

CASO $\mathrm{N}^{9}$ 4. - Osteoartritts del fémur $\mathrm{y}$ articulación coxofemoral derecha. Artritis de la rodilla derecha.

J. L. C. S. - Obs. N $49 / 57964$.

Se trata de un niño de 6 años que ingresa al hospltal el 15-VI-46 por una fiebre tifoidea que llevaba ya 16 días de evolución. El diagnóstico se confirmó mediante el hemocultivo.

clínlcarnente se trató de una tifoldea may grave. Este estado de gravedad se mantuvo durante 12 dias, plazo en el cual estuvo inconsciente, no se alimentaba o lo hacía en forma muy deficlente, el estado general era pésimo, presęntó supuración del oído derecho durante algunos días y hubo un franco compromiso del miocardio. En los días sucesivos fué mejorando paulatinamente, iniciándose el tratamiento con cloromicetina a los 14 días de hospitalización. La fiebre desapareció 4 dias más tarde. Pasa luego 19 dias afebril. A los 42 dias de hospitalización (26-VII-49) presenta nuevamente alzas térmjcas, está más decaido y a nivel de la rodilla derecha acusa dolor $y$ ligero aumento del calor local. Sigue luego un período febril de 25 días, en que la temperatura fluctuaba entre 38 y $39^{\circ}$. Dos dias más tarde hay también signos de inflamación a nivel de la región trocanterigna.

Al día siguiente se toman las radiografias Nos. 71:397-98-99, que fnforman: "No se observan leslones oseas".

A los 50 días de hospitalización se agrega impotencia funclonal del miembro inferior $y$ posictón plciosa en flexión de la pierna sobre el muslo y de éste sobre la pelvis. Hałia dolor en Ia parte interna de la interlínea articular e fntenso dolor en una zona del tamaño de un peso a nivel del cóndllo femoral derecho. Esta sintomatología regresó pronto, influída tal vez por la aceín de los antiblótjcos.

FI 5-VIII-49 se inicla el tratamiento con sulfatiazol y penicilina, colocándose en el plazo de 10 dias, $3.300,000$ U.

gl 12-VIII-49 acusa nuevamente ligero dolor a nivel de la rodilla derecha. la que se nota con los relieves anatómicos menos marcados. Una semana más tarde siente dolor a nivel de la cadera derecha. Existía fiebre desde 2 dias antes. Nuevamente adoptó el ni- 


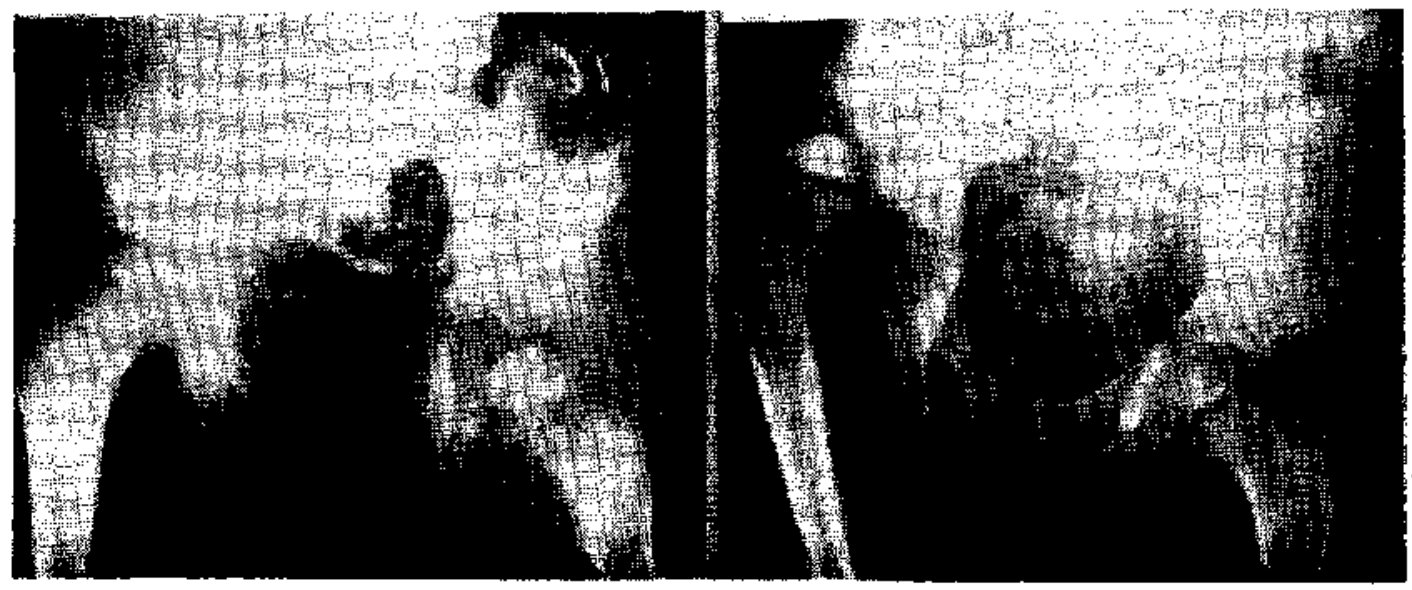

Radiografia N 72616: Se observa desenso del fémur y una diástasis del hueso con raspecto a la cavióad coti.oidea. Hay una mayor alteración ósea a nivel del cuello. Existe una formación ósea por encima de la cavidad cotiloídea.

ño una posición viciosa. A nivel de la cadera derecha se observaba una zona de infiltraclón muy dolorosa y con aumento de la temperatura local.

Se tomó la radiografía $N^{\circ} \mathbf{7 2 0 0 3}$, en la que se observa un proceso destructivo a nivel del cuello del fémur.

EI 20-VIII-49 se punciona la articulación, obteniéndose un líquido hemorrágico, cuyo cultivo resultó negativo.

El 7-IX-49 se toma la radiografia de control $N^{r:} 72282$, en la que sa observa un descenso apreciable del fémur. Una nueva radiografía (72016), tomada 16 días más tarde, muestra una reducción de la cabeza femoral; pero hay una gran diástasis respecto a la cavidad cotiloídea. Se coloca un yeso pelvipedio en abduceión máxima. No se logró una rotación interna suficiente, por existir rigidez marcada a nivel de la articulación y no se insistió con maniobras forzadas nor temor a la fractura a nivel del clello.

El 27-IX-49 se tcma la radiogralia No 72772 , en la que se apresla una reducción satisfactoria y se indica control en 3 meses.

Un año más tarde aun se mantenia el yeso por actividad del proceso (radlografía $\mathbf{N}^{\text {" 215). }}$

Resumen: Corresponde esta observaciớn a un niño que presentó una fiebre tifoidea muy grave, tratada con cloromicetina, que a los 60 días de haber comenzado, presentó una artritis de la rodilla derecha, que cedió pronto, tal vez por efecto de los antibióticos; pero que luego tuvo una artritis de la cadera derecha, con un proceso destructivo a nivel del
J. L. C. S. - Caso $N^{\circ} 4$.

Radiogtafia No 72003: Se aprecia un proceso de rarefacción del suallo femoral derecho y alteraciones de la estructura de la cortical interna. Además, hay una laxación patológica.

cuello femoral y una luxación patológica. No nos fué posible determinar la etiología exacta del proceso articular, ya

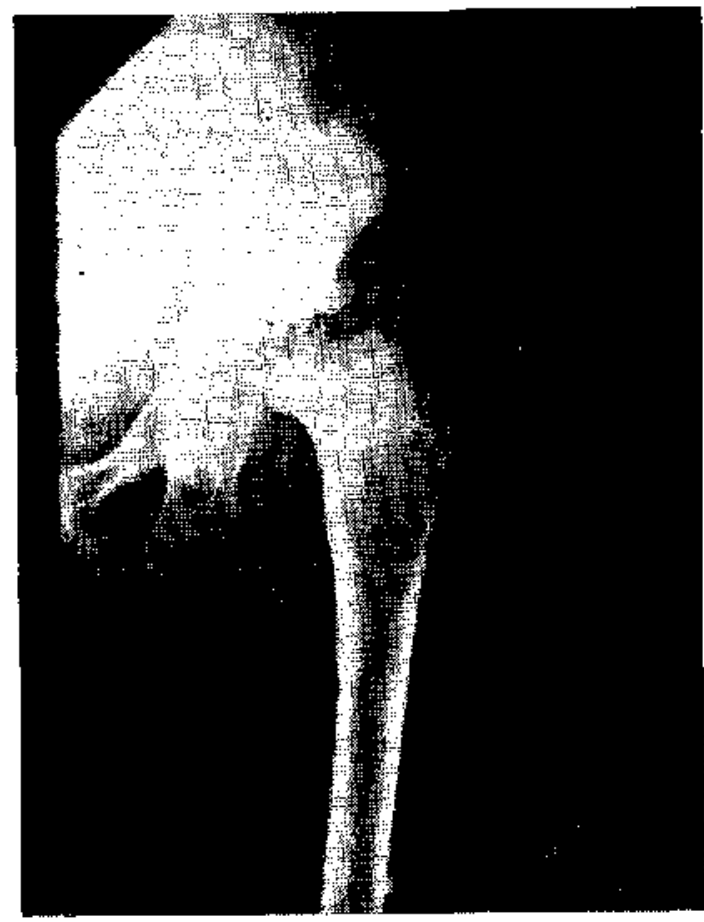

J. L. C. S. - Caso $\mathrm{N}^{*} 4$.

Radiografía № 215: Se ve una deatrucción del núcleo epifisiario proximal del fémur y una luxación patológica. Hay formaciones osteofiticas por encima de ia cavidad cotiloidea. 
que el líquido extraído por punción articular resultó estéril. Un hemocultivo hecho en el momento de iniciarse la artritis de la rodilla derecha desarrolló un estafilococo dorado hemolítico. Asociando estos hechos se puede pensar que éste fué el germen causal del proceso articular. La evolución fué muy arrastrada y después de un año de estar enyesado aun no hay evidencia de curación. Seguramente quedará con secuela.

CASO $\mathrm{N}^{*}$ 5. - Osteomielitis de la epiflsis superior del fémur derecho.

\section{N. C. A. - Obs. NP 4io/1180s.}

Infermo de 7 años que ingresa al hospital por una flebre tifojiea el 22-IX-46. La enfermedad actual se había 1niciado en los últimos dias de agosto. Ocho dias antes del ingreso se queja de dolores en el tríángulo de scarpa y muslo derechos, que le implden moverse y sentarse solo. En el momento del ingreso habia dolor al menor movimiento de la articulación coxofemoral derecha. También existía ictericia, además de las sintomas y signos propios de la tifoidea.

La evolución se caracterizó por una curva febril intermitente. La ictericia se notaba apenas a los 15 días de hospitalización. El dolor que el niño localizaba en un principio en la articulación coxofemoral derecha, desaparece totalmente al cabo de un mes. Se piensa, en vista de que tanto la sintomatología física como la radiológlca era negativa, que se trataba de un proceso de perlarteritis o de perlostitis, que no alcanzó a camprometer el hueso.

Permanece en cama sin sintomas durante 20 días más. A los $\mathbf{5 1}$ dias de hospitalizaclón se le autoriza levantarse. Nuevamente slente dolor a nivel del muslo derecho y la flẹtore reaparece, alcanzando hasta $39^{\circ}$. Se toma una rajiografía (57028) de la cadera y sé comprueba una asteomlelitis de la epifisis superior del fémur derecho, con coxa vara concomitante. El 22-XI-46 se coloca tracción hasta el 4-XII-46. Ese día se inmoviliza con yeso. El 14-II-47 se toma, una radiografía (58443), que revela persistencia de las lesiones del cuello del fémur. El 15-IX-47 aun no hay reparación ósea, por lo que se coloca nuevamente yeso. El 12-XII-47 se toma la radiografía $\mathbb{N}^{\circ}$ 62866, que revela una franca mejoria; vero la cadera tiene tendencia a la coxa vara. Deambula sin dificultad. El 17 de ese mes se coloca calzón de yeso. Permanese en estas condiclones durante 4 meses. In examen hecho en abrll de 1948 permite apreciar que el niño ha curado desde el punto de vista clinico. Subjetlvamente se siente blen. Se toma una radiografia de control, que muestra la existencia de una coxa vara.

Resumen: Este caso corresponde a una osteomielitis de la epífisis superior del fémur derecho con coxa vara concomitante. De esta observación se desprende la conveniencia de tomar una radiografía para descartar una lesión ósea o articular, cuando clínicamente hay fundamento para sospecharla. A pesar de haber estado 20 días sin sintomatología, bastó levantarlo para que ésta reapareciera. Esta complicación mejoró con tracción e inmovilización con yeso. Como secuela quedó una coxa vara.

CASO No 6. - Artritís sacrolljaca derecha.

\section{J. V. I. - Obss. No $47 / 29617$.}

Se trataba de un niño de 11 años de edad, que ingresa al hospital el 28-vII-47 por una fiebre tifoidea confírmata, que llevaba 24 días de evoluctón. Una semana antes de consultar $y$ en concomitancia con fiebre alta y mantenfda, apareció un dolor intenso y continuo, ubicado especialmente en la región de la cresta llíaca derecha. Este dolor se exacervaba con los movimientos y se irradiaba hasta el talón. El examen físico de ingreso mostró, además de la hépato y esplenomegalla, proplas de la fiebre tlfoidea, dolor difuso a lo largo de la extremidad inferior derecha $y$ que era más acentuado en la parte proximal del miembro. Al tercer dia de hospitalización existia dolor exquisito en el borde posterior del trocánter mayor. Tamblén había dolor intenso al movilizar la articulaclón coxo femoral y al palpar suayemente cualquier punto cercano a ella. Se nótaba, además, tolor de mediana intensidad en la parte baja del muslo $y$ alta de la plerna y escaso dolor en la parte distal de la extremidad. Al $80^{\circ}$ dia de hospitalización se precisa una zona intensamente dolorosa en la articulación sacroilíaca derecha. Persistia siempre el dolor en la cara pasterior del trocánter mayor. En los dias sucesivos fué disminuyendo el dolor y ya a los 20 dias estaba en condlciones de camlnar, pero claudicando. Posteriormente también pasó esto y se dió de alta en buenas condiciones. Fi 27-VIII-47, a los 31 dias de hospitalización y 55 de enfermedad, se toma una radtografía cuyo informe fue el siguiente: "Ia articulación sacrolliaca derecha se observa de limites difusos. Hay una pequeña zona de osteoesclerosis en el bla derecha tel sacro".

Resumen: Esta observación corresponde a una fiebre tifoidea, que a los 32 días de evolución se complicó con una artritis 
de la articulación sacroilíaca derecha y que evolucionó en el plazo de 3 semanas. La radiografía mostró, además, la existencia de una pequeña zona de osteoesclerosis en el ala derecha del sacro.

CASO N 7 . - Periostitis de la tibla izquierda.

G. M. M. - Obs. N $\mathbb{N}^{1 / 4} 8734$.

Se refiere a un enfermo de 10 años, que ingresa al hospital por una fiebre tifoidea conf1rmada, que llevaba 7 dias de evolución. A Ios 49 dias de enfermedad se observa un aumento de volumen muy doloraso en el borde interno del $1 / 3$ inferior de la tibia. En este sitlo existia ligera rubicundez. Cuatro días más tande se observa una tumefacción del tamaño de una moneda de un peso en la zona antes descrita. En los días sucesivos desaparecieron los signos de. Inflamaclón, quedando sólo el aumento de volumen indoloro en la regićn tibial. Se tomó la radiografia $N^{\prime} 47318$, que reveló una periostitis.
Resumen: Se trató en el presente caso de una fiebre tifoidea de mediana gravedad en un niño de 10 años y que en plena convalecencia presenta como complicación una periostitis de la tibia izquierda y cuya sintomatología regresó rápidamente.

\section{CASO NN 8.}

R. M. A. - Obs. N" 71811 .

Corresponde a un enfermo de 12 años, que ingresa al hospital por una fiebre tifoldea confirmada, el 9-XII-43. Llevaba en el momento del ingreso 3 dias de evolución. Se trato de una fiebre tifoidea de mediana gravedad, que se mantuvo febril durante 31 dias. En el curso de su hospitalización presentó abscesos múltiples del cuero cabelludo. A los 22 dias de hospltaltzación se aprecia un panadizo a nivel del pulgar izquilerdo, que se vacia, dando salfda a escaso pus y se procede a

Algunas caracteristicas de los 8 enfermos de fiebre tifoidea que presentaron complieaciones osteoarticulares.

\begin{tabular}{|c|c|c|c|c|c|c|c|c|c|c|c|c|}
\hline \multirow{4}{*}{ abs. $\mathbf{x}^{0}$} & \multirow{4}{*}{ 氧 } & \multirow{4}{*}{ 㝵 } & \multirow{4}{*}{ 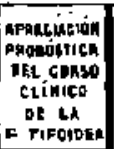 } & \multirow{4}{*}{ 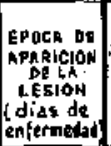 } & \multirow{4}{*}{$\begin{array}{c}\text { TIPO } \\
\text { DE } \\
\text { LA } \\
\text { LESION }\end{array}$} & \multirow{4}{*}{$\begin{array}{l}\text { LOCALI- } \\
\text { XACIOA } \\
\text { OF LA } \\
\text { LEgION }\end{array}$} & \multicolumn{6}{|c|}{ SINTOMATOLOSIA Y EVOLUCIÓN CLINICA } \\
\hline & & & & & & & \multicolumn{2}{|c|}{ GENEAALL } & \multicolumn{4}{|c|}{ LOCAL } \\
\hline & & & & & & & \multirow{2}{*}{$\begin{array}{l}\text { compno } \\
\text { Giso } \\
\text { Getren }\end{array}$} & \multirow{2}{*}{ Fifiane } & \multicolumn{2}{|c|}{ SUDJETIVOS . } & \multirow{2}{*}{ osjerivos } & \multirow{2}{*}{ MEGRt: } \\
\hline & & & & & & & & & DOLOR & Aescresion & & \\
\hline 1. T. O. & 13 & $r$ & Grave & 66 & $\begin{array}{l}\text { Espandi } \\
\text { litis }\end{array}$ & $\mid \begin{array}{c}11, y 12 \\
\text { verteporas } \\
\text { aromsales }\end{array}$ & Rcentus & $39^{\circ}$ & 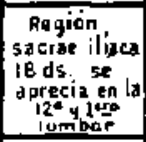 & dias & 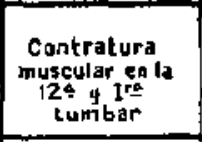 & $3 \mathrm{~m} .25 \mathrm{ds}$. \\
\hline $\begin{cases}5 . \text { A. A. } \\
+0 / 50637\end{cases}$ & 12 &.$M$ & gante & 34 & $\begin{array}{c}\text { Osteonar- } \\
\text { tritis }\end{array}$ & Mriperd & Wo hubo & $\left\{\begin{array}{l}\text { Periodo } \\
\text { drffi: } \\
\text { boliteo }\end{array}\right.$ & $\begin{array}{l}\text { Muneca } \\
\text { izquierda }\end{array}$ & $\begin{array}{l}55 \\
\text { dias }\end{array}$ & $\begin{array}{l}\text { Calor local } \\
\text { enrojecimiento } \\
\text { de rol.impotentia } \\
\text { funcional }\end{array}$ & 48 dias \\
\hline $\begin{array}{lll}5 . & \text { E. A. A } \\
46,12206\end{array}$ & 6 & $\mathbf{F}$ & GAMVE & 15 & $\begin{array}{c}\text { Osteoar- } \\
\text { trit is }\end{array}$ & $\begin{array}{c}\text { Fémur } \\
\text { y } \\
\text { art., coto } \\
\text { femoral } \\
\text { ixqda. }\end{array}$ & $\begin{array}{l}\text { hasn. } \\
\text { twasdo }\end{array}$ & $\begin{array}{c}\text { Propia } \\
\text { de ia } \\
\text { onter } \\
\text { medid } \\
390\end{array}$ & $\begin{array}{l}\text { Art. coxo- } \\
\text { femoral y } \\
\text { tibiotersinat } \\
\text { isquierda }\end{array}$ & $\operatorname{dias}^{14}$ & 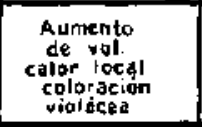 & 45 dias \\
\hline $\begin{array}{l}A, C . A . \\
46 / 1\end{array}$ & 7 & M] & CAAYR & 14 & $\begin{array}{c}\text { Osteomie } \\
\text { litis }\end{array}$ & $\mid \begin{array}{c}\text { Femur } \\
\text { oerecho }\end{array}$ & $\begin{array}{l}\text { Propio } \\
\text { de la } \\
\text { enfer- } \\
\text { medad }\end{array}$ & $\begin{array}{l}\text { Propia } \\
\text { de id } \\
\text { dnfifine } \\
\text { ded } \\
39^{\circ}\end{array}$ & $\begin{array}{l}\text { A de Suarpa } \\
y \text { mus } 10 \\
\text { derecho }\end{array}$ & $38 \mathrm{ds}$. & 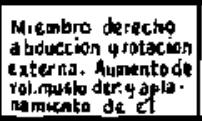 & \\
\hline ]. L. $C$ & 6 & M & GAAVE & $\frac{58}{60}$ & 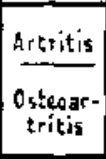 & 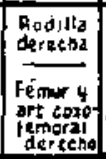 & $\begin{array}{l}\text { Acken } \\
\text { tudda }\end{array}$ & $40^{\circ}$ & 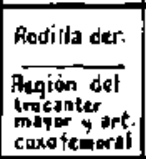 & $\begin{array}{l}16 \text { ds. } \\
\text { lano }\end{array}$ & 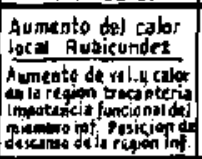 & \begin{tabular}{|c|}
$16 \mathrm{ds}$. \\
$14 \mathrm{ds}$.
\end{tabular} \\
\hline $\begin{array}{l}\text { J. V. } 1 \\
47 / 22617\end{array}$ & 11 & $\mathbf{M}$ & $\begin{array}{l}\text { MEDIAHA } \\
\text { GRAYYE DAD }\end{array}$ & 17 & $\begin{array}{l}\text { Ostemoar- } \\
\text { tritis }\end{array}$ & $\begin{array}{c}\text { 5acro } \\
\text { Art.sacto } \\
\text { iliaca } \\
\text { derecho }\end{array}$ & $\begin{array}{c}\text { Prapia } \\
\text { de Ia } \\
\text { enfer- } \\
\text { medad }\end{array}$ & $375^{\circ}$ & 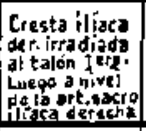 & $27 \mathrm{ds}$ & No se observo & \\
\hline G. M. M. & 10 & $|d|$ & $\begin{array}{l}\text { MEDSAMA } \\
\text { GRAYGDAO }\end{array}$ & 5 & $\begin{array}{c}\text { Parios. } \\
\text { titis }\end{array}$ & Tirquia & No hubo & $37^{\circ}$ & $\begin{array}{l}\text { Borde interno } \\
\text { da latitibia } \\
\text { reqgian inf. }\end{array}$ & 8dias & $\begin{array}{l}\text { Aumento de val. } \\
\text { tercio inf de la } \\
\text { pierno iequierda } \\
\text { Rubicundex }\end{array}$ & Bdisc \\
\hline $\begin{array}{l}\mathrm{R}, \mathrm{W}, \mathrm{M} \\
4 \%\end{array}$ & 12 & $M$ & $\begin{array}{l}\text { MEDIAMA } \\
\text { GRAYEDAD }\end{array}$ & 27 & Osteitis & $\left|\begin{array}{c}\text { Pulgar } \\
\text { izad. } \\
\text { (tolenge } \\
\text { distal })\end{array}\right|$ & Wo hu bo & $40^{\circ}$ & $\begin{array}{l}\text { Falange } \\
\text { distaj } \\
\text { pulgur izq. }\end{array}$ & $27 \mathrm{ds}$. & $\begin{array}{l}\text { Dolor - calor. } \\
\text { mutmanto de volu. } \\
\text { men. Campio de } \\
\text { calocación. }\end{array}$ & 27 dias \\
\hline
\end{tabular}


extraer la uña. Se encontró un trayecto fistuloso profundo, con exposición de la falange ósea. El pus dió desarrollo a estafílococo dorado hemolitico. Ocho dias más tarde se infeia el tratamiento con sulfatiazol, a razón de $3 \mathrm{~g}$ dlarios, durante 12 días. A los 45 días de hospitalización se toma la radiografia $N^{9} 42094$, que informa: "Osteitis de la falange distal. Desprentimiento epifisiario". Ese día se extrajo fácllmente la falange. Cuatro días más tarde ya no se aprecla supuractón a nivel del pulgar izquierdo $\mathrm{y}$ la herida cicatriza en buenas condiciones.

Resumen: Se trata en este caso de una fiebre tifoidea que en el curso de su evolución presentó numerosos abscesos del cuero cabelludo y de donde procedieron seguramente los gérmenes que originaron la osteítis de la falange distal del pulgar izquierdo, complicación que sólo vino a mejorar cuando se extrajo este segmento.

En el cuadro anterior se resumen las características de mayor interés observadas en los 8 enfermos de fiebre tifoidea que presentaron complicaciones osteoarticulares con lesiones radiológicas.

\section{Resumen y comentario.}

En el cuadro pueden observarse algunas de las características más importantes que presentaron nuestros enfermos. Como puede verse, la edad fluctuó entre 6 y 12 años. El compromiso esquelético pudo demostrarse generalmente pasada la segunda semana de enfermedad, apareciendo las lesiones en períodos oscilantes entre 5 y 66 dias a partir del comienzo de la afección. El curso clínico de la tifoidea fué calificado en 5 ocasiones como grave $y$ en 3 como de mediana gravedad. En los casos calificados como graves, hubo en 3 , además de la complicación ósea, varias otras. El tipo de la lesión, así como su localización, fué variable: así hubo una espondilitis de la región dorsal; 1 osteoartritis de la muñeca izquierda; 2 osteoartritis de la articulación coxofemoral y fémur; 1 osteomielitis del fémur derecho; 1 osteítis del sacro y de la articulación sacroilíaca derecha; 1 periostitis de la tibia izquierda $\mathrm{y}$, finalmente, una osteitis de la fa- lange distal del pulgar izquierdo. La sintomatología se tradujo en algunos casos en gran compromiso del estado general y fiebre; en otros, en cambio, sólo se apreciaron fenómenos locales, consistentes en dolor y alteraciones de tipo inflamatorio, como tumefacción, etc. En general, se observó una rápida regresión del dolor, salvo 1 caso de artritis de la cadera, que lo presenta un año después de la lesión y pese a la inmovilización que se le ha impuesto. En cuanto a los sintomas objetivos regresaron a veces antes que el dolor, y otras, después. Las lesiones radiológicas correspondieron a aquellas que se observan en cualquier proceso óseo o articular de origen inflamatorio. En la mitad de los casas se observó Ieucocitosis; los demás tuvieron un recuento normal. La sedimentación estuvo alterada, a veces con valores muy elevados; pero es necesario considerar que muchos casos se presentaron en plena convalecencia de la fiebre tifoidea $y$ entonces ésta es de por sí elevada. Sólo se observaron secuelas en los casos de osteoartritis de la cadera, consistentes en acortamiento del miembro o coxa vara y en el caso de la espondilitis, que quedó con una pequeña cifosis.

En un enfermo se logró aislar el B. de Eberth mediante la punción de la articulación afectada (osteoartritis de la muñeca).

El tratamiento consistió en inmovilización, especialmente con yeso; en tracción, cuando el caso así lo requería y en la administración de antibióticos, cuando estuvieron disponibles.

No sabemos hasta qué punto la administración de cloromicetína sexá capaz de suprimir las alteraciones óseas descritas, ni tampoco el efecto que tendrá el fármaco en la lesión ya instalada. Recientemente, Traisman y Champlin ${ }^{10}$ han pubiicado un caso de osteomielitis con localizaciones múltiples producida por una Salmonella Typhimurium, que respondió al tratamiento con cloromicetina. Por otra parte, Morse y Geiser ${ }^{6}$ tratan con éxito un caso de complicación ósea producido por el $B$. de Eberth. 
Meneghello y cols.", en 100 niños con fiepre thioidea tratados con cloromicetina, solo observarọn 1 entermo con complicacion osteoarticuiar. corresponde al caso $N \div 4$ de nuestra casuistica.

Otro aspecto que vale la pena comentar, es el de un grupo de 9 niños, que en forma brusca experimentaron un vivo $y$ violento dolor, sea a nivel de una costllla o de un hueso de las extremidades. En algunas oportunidades, este dolor, que estaba localizado en un punto preciso, se acompañaba de ligero aumento de volumen circunscrito. $\mathrm{Ni}$ la administración de aspirina ni otros calmantes ejercían el menor efecto. Hubo veces en que fué necesario recurrir a la morfina. Él cuadro pasó en general, en forma rápida $\mathrm{y}$ el estudio radiológico no logró demostrar lesión ósea. Creemos que estos casos pueden corresponder a osteítis mímimas, que no alcanzan a visualizarse a rayos.

Finalmente, ha habido un grupo de 6 enfermos con manifestaciones típicas de artritis o de osteítis; pero en los cuales el estudio radiológico mostró una indemnidad del hueso o de la articulación estudiada.

\section{Conclusiones.}

1) Las manifestaciones osteoarticulares con lesiones radiológicas son raras en la. fiebre tifoidea. En nuestra casuística se presentaron en el $1.16 \%$ de los casos.

2) En general este tipo de alteraciones aparece más allá de la tercera semana de evolución de cuadros tificos graves o de mediana gravedad.

3) En muchas ocasiones no existe un notorio compromiso general.

4) Las alteracjones locales regresan a menudo en forma arrastrada.

5) En cuatro ocasiones apreciamos secuelas que correspondieron a fenómenos de cifosis, coxa vara y acortamiento del miembro.

6) El tratamiento actual consiste esencialmente en inmovilización ortopédica, a menos que se demuestre acción de los nuevos antibióticos.

7) Seis enfermos tuvieron manifestaciones típicas de osteítis o de artritis, re- velando el estudio radiológico indemnidad del hueso o articulación estudiada.

8) En 9 enfermos se observaron manifestaciones dolorosas violentas y bruscas, en relación tal vez con osteitis mínimas.

\section{Summary.}

Osteoarticular manifestations with radiological lesions are rare in typhoid fever. The authors found them only in $1.16 \%$ of their cases.

These lesions generally appear after the third week in severe or moderatly severe cases and in many cases without a marked general involvement.

The local lesions regress slowly. In 4 cases there remained as secuelae kyphosis, coxa vara and shortening of an extremity. Present day treatment consists oi ortnopaedic inmopilization unless good results are demonstrated with the newer antibiotics. Six patients had typical manifestations of osteitis or arthritis with negative $X$ ray studies. Nine patients had severe joint pains of short duration probably aue to very minute foci of osteitis.

\section{Bibliografía.}

1.-ABBASSY SHAFIK - Mielitis compresiva debida a un absceso tifico subperiostal. Arch. of Ped. 63: 63, 1946.

2.-ABT, J. y ABT, A. - Brenneman. Tomo 2, cap. 30, pág. 17.

3.-AILENDE, G. Y ZARAZAGA, J. - Observaciones de 6 casos de espondilitis tifica. Rev. de Ortopedia y Traumatología 6: $89,1946$.

4.- HaRRIES y MITMAN. - Práctica clínica de las enfermedades infeceiosas. Pág. 442. Editorial Miguel servet, Madrid-Barcelona, 1943.

כ.-MFNEGHELLO, J.; UNDURRAGA, o.; GAILO, A. y RAIMANN, A. - Tratamiento de 100 casos de fiebre tifoldea on el riño con cidrarafenicol (cloromicetina). Rev. Méd. de Ĉ́hile 89: 307, 19:511.

6.-MORSE, CH. y GETSER, F. - Arch. Int. Med. 85: 280, 1950 .

7.-MINOR, G. y WHITE, L. - Ann. Int. Med. 24: 29,1946 .

8.-NOTTI, P. y MIYARA, S. - Rev. de la Asoc. Méd, Arg. 81: 125, 1947.

9.-PIIOD, M. - Encriclopédie Médico Ch!rurgicale. Capítulo 8021, pág. 12.

10.-TRAISMLAN, H. y CHAMPLTN, G. - Jour. of Ped. 38: 244, 1851 .

11.-JAUREGUY, M. y SEMON, G. - Arch. de Ped. del Uruguay 18: 298, 1947. 